\title{
Clinical interpretations of the effectiveness of changes in body position during aerobic fitness after neurologic injury
}

\begin{abstract}
Nur Fariza Izan ${ }^{1}$, Sheikh Hussain Salleh ${ }^{1,2,3}$, Chee-Ming Ting ${ }^{1,4}$, Fuad Noman ${ }^{1}$, Hadrina Sh-Hussain ${ }^{1, *}$, Roman R. Poznanski ${ }^{5}$
\end{abstract} and Ahmad Zubaidi Abdul Latif ${ }^{6}$

\author{
${ }^{1}$ School of Biomedical Engineering and Health Science, Faculty of Engineering, Universiti Teknologi Malaysia, 81310 , \\ Johor, Malaysia \\ ${ }^{2}$ Centre for Biomedical engineering, Universiti Teknologi Malaysia, 81310 , Johor, Malaysia \\ ${ }^{3}$ Health Education and Lifestyle (HEAL ULTRA), 81300 , Johor, Malaysia \\ ${ }^{4}$ School of Information Technology, Monash University Malaysia, 47500, Bandar Sunway, Malaysia \\ ${ }^{5}$ Faculty of Computing and Informatics, Universiti Sultan Zainal Abidin, 22200, Beset, Terengganu, Malaysia \\ ${ }^{6}$ Neurosurgery, Faculty of Medicine, Universiti Sultan Zainal Abidin, Medical Campus, 20400, Kuala Terengganu, \\ Terengganu, Malaysia
}

${ }^{*}$ Correspondence: sh.hussain@tutanota.com (Hadrina Sh-Hussain)

DOI: $10.31083 /$ i.jin.2020.03.222

This is an open access article under the CC BY 4.0 license (https://creativecommons.org/licenses/by/4.0/).

The purpose is to estimate the effectiveness of electrocardiograms during resting and active participation by the differentiation between the electrical activity of the heart while standing and sitting in a resting state. The concern is to identify the electrocardiogram parameters that did not show significant changes within these positions. The electrocardiogram parameters can be considered to be a standard marker for medically compromised patients. The electrocardiogram is recorded in the standing and sitting positions focusing on healthy participants using standard electrode placement of lead-I. Combined lead-I patterns (camel-hump or ST-segment prolongation) are usually seen in neurologic injury or hypothermia patients. The pairwise comparisons of a year data are about 454,400 cycles of sitting and 493,470 cycles of standing data. Thus, it is essential to quantify the nature and magnitude of changes seen in the electrocardiogram with a change of posture from sitting to standing in a healthy individual. This makes the findings of electrocardiogram analysis in this paper interesting in which some parameters (i.e., camel-hump patterns in lead-I) are helpful for clinical interpretations and could be suggestive of neurologic injury.

\section{Keywords}

ECG; aerobic exercise; standing position; sitting; fiducial points; ANOVA; neurologic injury

\section{Introduction}

Electrocardiogram (ECG) is the most common non-invasive method used for various conditions of heart diagnosis. The ECG is also used extensively for monitoring patients, especially in the
Intensive Care Unit (ICU), emergency room, and in the operation theaters during surgical procedures. Various diagnostic tests, such as exercise stress tests, also monitor ECG changes to trace the underlying cardiac pathologies (Khare and Chawala, 2016). The standard procedure for recording the ECG is in the supine position. The values of various parameters that define normality are based on calculations made from supine ECG recordings. However, in certain situations, such as exercise stress testing and evaluation of syncope by the head-up tilt test, the ECG is recorded in the upright position. The same may also be necessary for patients who cannot assume the supine position due to certain conditions that require them to adopt other postures.

Most of the previous studies have focused on comparing the ECG recorded in the supine with the left and right lateral positions in patients being monitored for myocardial ischemia in the ICU (Adams and Drew, 1997). Khare and Chawala (2016), on the other hand, has started a study on the effect of a change in the supine and standing positions on the mean frontal plane QRS vector, P-wave, T-wave, amplitude of P, QRS, and T waves, ST-segment changes, and QTc interval. The mean frontal plane QRS axis recorded in the supine and standing positions was comparable. The reclining and sitting ECG shows a significant variation of the mean QRS axis as compared to the supine position. The T-wave axis was found to be comparable in the supine and standing positions. The QTc interval showed a significant change with a change in the body position from supine to standing.

It is also equally important to study the magnitude of changes seen in the ECG with a sitting and standing position in healthy individuals. The results would be useful to the interpretation of ECG recorded during exercise stress tests. It would also provide an insight into the feasibility of recording the ECG in the seated position in patients being examined in the outpatient department of 
hospitals. This study was carried out to describe the changes in 26 parameters of ECG in terms of the mean amplitude of waveforms, the intervals, the segments, and the slopes on the position of sitting and standing.

Detection of ECG fiducial points can be defined as the automatic temporal localization of ECG waveforms (P-wave, QRS, and T-wave) onset, peak, and offset points. Detection of the QRS complex has been extensively investigated in the literature, yet there is no universal solution to cover the diversity of QRS waveforms. In contrast, fewer studies have been carried out on the detection of $\mathrm{P}$ and $\mathrm{T}$ waves, due to the complexity of detecting such waves in unusual and noisy cases. Systematic literature reviews over the past three decades of QRS detection methods are conducted by Elgendi et al. (2014); Kohler et al. (2002); and Martis et al. (2013). The typical proposed methods can be categorized into several types: (i) derivatives and digital filtering methods (CastellsRufas and Carrabina, 2015; Chatterjee et al., 2012; Hamilton and Tompkins, 1986; Karimipour and Homaeinezhad, 2014; Ning and Selesnick, 2013; Pan and Tompkins, 1985; Phukpattaranont, 2015), (ii) transformation-based methods (Bono et al., 2014; Ieong et al., 2014; Madeiro et al., 2013; Martínez et al., 2010; Merah et al., 2015; Pal and Mitra, 2012; Yochum et al., 2016; Zhu and Dong, 2013; Zidelmal et al., 2014), (iii) machine learning methods (Akhbari et al., 2018, 2016; Lin et al., 2010) and (iv) other methods (curve fitting (Tafreshi et al., 2014), mathematical modeling (Madeiro et al., 2013), template matching (Bashir et al., 2014; Chen and Chuang, 2017), correlation analysis (Homaeinezhad et al., 2014; Karimipour and Homaeinezhad, 2014), point process tracking (Citi et al., 2012), power analysis (Kim and Shin, 2016), and masking \& amplitude analysis (Chen and Chuang, 2017).

The derivative and digital filters are the most widely used ECG delineation methods due to their less complex, and they do not require high computational resources. One of the most well-known methods for QRS detection is the Pan-Tompkins method (Pan and Tompkins, 1985) and its update (Hamilton and Tompkins, 1986). These methods are commonly used to delineate normal ECG signals and mostly fails to process the abnormal ECG signals. The Wavelet transform (WT) based algorithm also has been used for ECG delineation due to their suitability to locate different waves with typical time and frequency characteristics. However, WTbased methods still suffer from some significant drawbacks: prior information about the waveforms and their width is required, and WT uses very rigid arbitrary thresholds to determine the various wave components.

This work aims to evaluate whether the ECG recording alters the various components of the ECG curve after the change in neurologically injured patient's position during aerobic fitness. The ECG was recorded in the sitting and standing positions from one subject for one year using single-lead standard electrode placement (the three limb leads of Einthoven's triangle, left-arm, right-arm, and left-leg). We propose an automatic approach to extract a total of 26 temporal and morphological ECG parameters to be used for the statistical tests and analysis. The analysis in this study also aims to explore and identify various ECG parameters that have the potential to be set as markers for tracking health performance. The analysis of variance (ANOVA) statistical test is used to analyze and determine the existence of statistically significant differences between the extracted ECG parameters in a resting state for both sitting and standing positions.

\section{Methodology \\ 2.1 Database}

The CBE Analyzer device is used to collect the ECG data with a standing or sitting position during the resting state. The CBE Analyzer's stethoscope was placed on the subject's chest, at the tricuspid area for heart sound (HS) recording, and ECG electrodes were clamped to the left forearm, right forearm, and to the left leg that is the bipolar limb lead-I. Two bio-signals were recorded simultaneously for 1 minute and saved into a computer by using CBE Analyzer's application interface. The data are saved in wave pulse-code modulation (PCM) with a resolution of 16 bit and 8 $\mathrm{kHz}$ sampling rate.

The data acquisition system is capable of capturing and storing the raw data to the computer terminal. The overall gain for the ECG amplifier is 1000 with an effective filter. The module includes the right-leg drive for $50 \mathrm{~Hz}$ power-line suppression as well as a return path for the ECG. The module also contains an isolation amplifier and an isolated power supply to ensure safety and avoid high current leakage to the patient body. Fig. 1 illustrates the ECG and hear-sound components. To date, this study has collected 947870 cycles of resting data within a period of 1 year, starting from March 2014 to April 2015. However, due to the non-activity of the subject under study, the data was not collected for June and July 2014. Table 1 summarizes the data collection of resting-state with an almost balanced total number of cardiac cycles for sitting and standing positions.

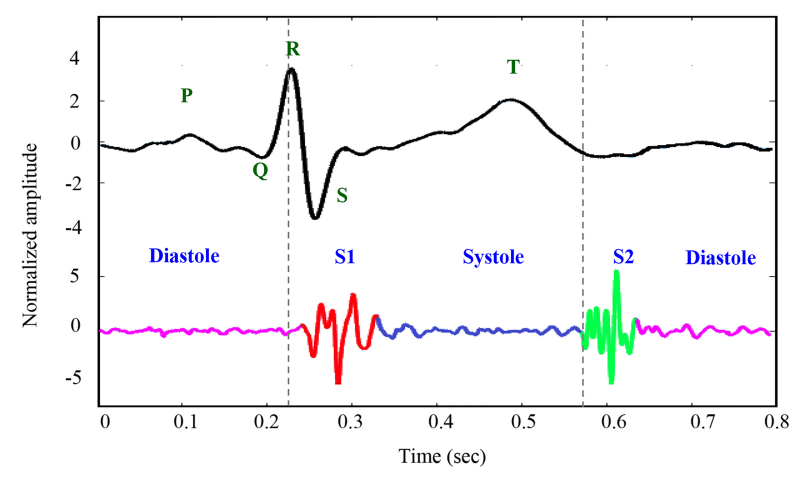

Fig. 1. Example of a single cardiac cycle consisting of ECG and heartsound signals with their components. The figure demonstrates the main components of the ECG signal (T-wave, QRS complex, and T-wave) and the fundamental sounds in the heart sound signal (S1, systole, S2, and diastole). The figure also illustrates the temporal alignment of cardiac electromechanical events where the S1 sound is aligned with R-peak, and S2 sound starts at T-wave end.

The ECG data were recorded simultaneously with HS signals to correlate the heart activity domains, where ECG represents the electrical activity, and the HS represents the mechanical activity. However, this study mainly focuses on the ECG data to understand if changes in subject position will alter the various components of the ECG signals. 


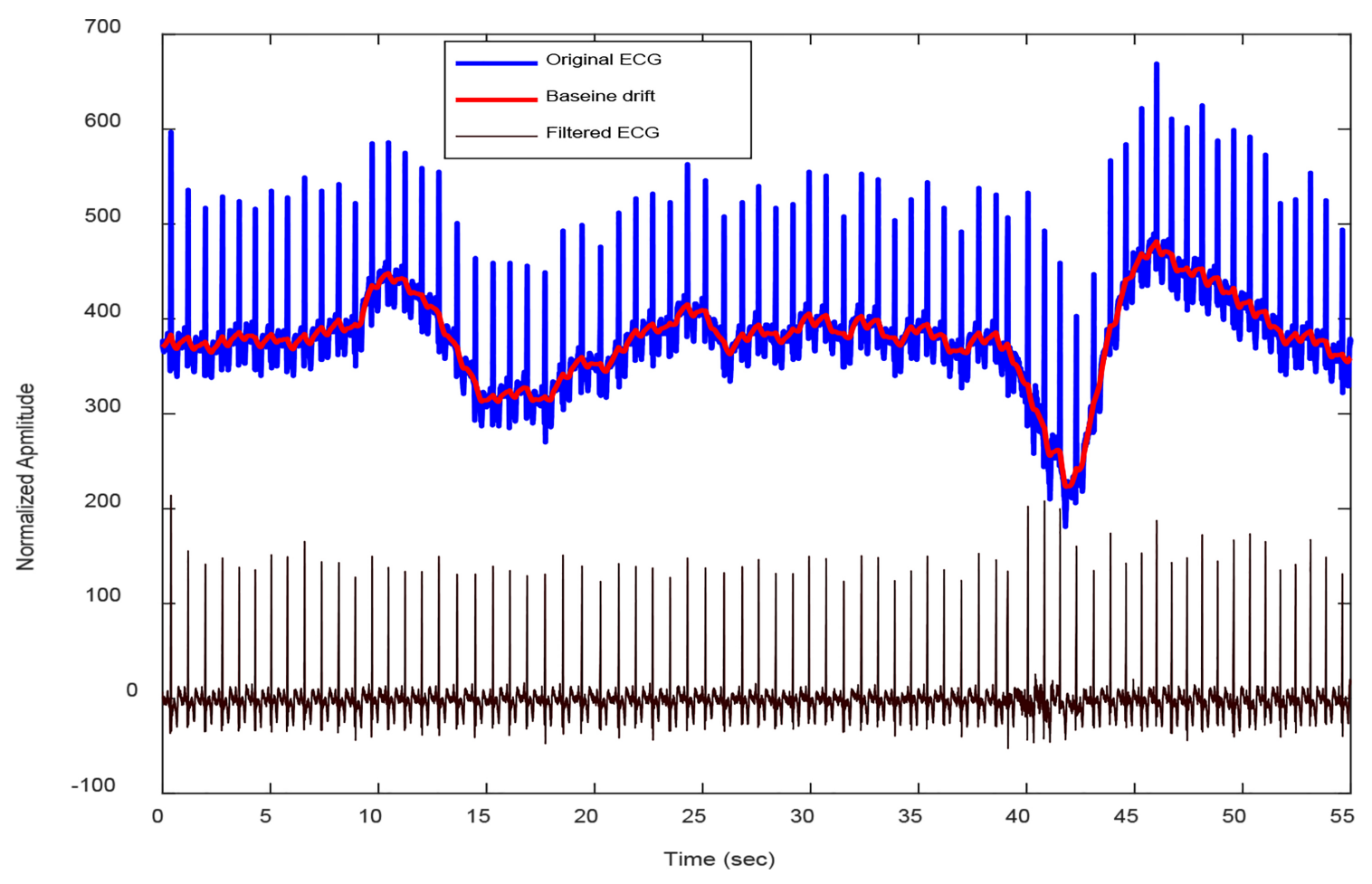

Fig. 2. Baseline drift removal using a median filter.In ECG automatic analysis procedure, and to reduce the complexity of signal processing, it requires removing the baseline drift and align the signal to the zero/dc line. The figure depicts the effects of filtering an ECG with severe baseline drift and how the signal is straightened, which make the R-peak detection much more straightforward.

Table 1. Details of data collection.

\begin{tabular}{lcc}
\hline Resting Data & Total cycles & Total ECG parameters \\
\hline Sitting & 17,476 & 454,400 \\
Standing & 18,979 & 493,470 \\
\hline
\end{tabular}

\subsection{Preprocessing}

In clinical practice, the ECG signals are usually contaminated by heterogenous noise sources, which degrade the signal quality. The predominant two types of noise that could be contained in ECG signals are baseline drift and powerline noise. Fig. 2 shows an example of an ECG signal with baseline drift noise. A Butterworth High-Pass filter with a cut-off frequency of $0.7 \mathrm{~Hz}$ is used to remove the baseline drift. A windowed median approach of digital subtraction filter is used to remove the high-power line frequency noise. According to Duskalov et al. (1998) and Levkov et al. (2005), this filter can preserve the spectral content and amplitude of the processed ECG signal.

\subsection{ECG Delineation}

A stationary wavelet transform is employed to denoise the ECG signal before the QRS detection procedure. The noisecontaminated ECG signal is decomposed with a three-level decomposition 'sym4'. The 'sym4' wavelet resembles the QRS complex. It attenuates the other ECG waves and emphasizes the QRS, which makes it an excellent choice for QRS detection. The ECG signal then undergoes a baseline drift filter, as detailed in Section 2.2. Sliding non-overlap windows of length $8 \mathrm{~ms}$ were used for tangent calculation. This reduces the amount of data to be pro- cessed to 1/8 of ECG signal length and speeds up the detection process. The tangent points are then analyzed, selecting only the points that reflect the concave and convex (crests and troughs) deviations of the ECG signal. The selected tangent points and the ECG signal are then used for the QRS detection process, which is initialized by setting two independent thresholds, a slope-based threshold:

$$
\gamma_{1}=\frac{1}{2 N} \sum_{i=1}^{N} \Delta_{i} \quad, \quad N=4
$$

where each $\Delta$ represents the $\mathrm{N}$ different maxima slope. This threshold is updated for each QRS during the detection procedure. The second threshold is amplitude-based and also adaptive. It was determined by selecting four maxima slopes from different intervals (1Fs, 2Fs, 3Fs, and 4Fs) considering that one of these maxima points $P k_{i}$ would represent an actual QRS peak amplitude:

$$
\gamma_{2}=0.7\left(\frac{1}{N} \sum_{i=1}^{N} P k_{i}\right)-\beta \quad, N=4
$$

where $\beta$ is an RR duration factor that is used in case, there is a sudden change in the heart rate (HR) (e.g., the tachycardia and bradycardia cases). The detection of the QRS peak is considered as the ECG amplitude that satisfies the thresholds values.

The detected QRS delineation points are used as a reference for the detection of the $\mathrm{P}$ and $\mathrm{T}$ wave. The intervals between each successive pair of QRS complexes (QRS offset to the subsequent 
QRS onset) are extracted from the original ECG signal. The filtering method for the QRS is different from the P-T waves, in which a zero-phase IIR high-pass filter is used to remove the baseline drift while maintaining P-T search regions. An important contribution of this algorithm, this filter removes the baseline drift of the ECG signals without introducing any deformation of the signal, thus avoiding the loss of any clinical information. The filtered signal is then passed through an average smoother, where each data point is sequentially replaced by the average value of adjacent points in sliding window spans. The smoothing is given by

$$
\begin{array}{r}
y(i)=\frac{1}{2 N+1}(y(i+N)+y(i+N-1) \\
+\cdots+y(i-N))
\end{array}
$$

where $\mathrm{y}(\mathrm{i})$ is the value of the $\mathrm{i}$ smoothed data point, $\mathrm{N}$ is the total number of data points present on either side of $y(i)$, and $(2 \mathrm{~N}+1)$ is the span.

The interval between each adjacent R-peaks was divided into two search blocks for $\mathrm{P}$ and $\mathrm{T}$ wave detection. The T-wave search block is initially defined as $60 \%$ (minimum $45 \%$ ) of the RR interval, which is then automatically adjusted based on the characteristics of concave and convex curves within this interval. The $\mathrm{P}$ wave search block begins one curve before the end of the $\mathrm{T}$ wave search block and ends at the subsequent QRS onset constrained to $25 \%$ of $\mathrm{RR}$ interval. A similar approach of QRS detection is implemented for $\mathrm{P}$ and $\mathrm{T}$ wave peaks detection using the heuristic analysis of maxima/minima points of tangent calculations.

Once the locations for the R, P, and T peaks were detected, the onsets and offsets of each wave were then located by defining search blocks around each peak. Initial locations of peaks onset and offset were found by testing the minima (location and amplitude), and then the tangent is used to investigate the availability of split waveform morphology. Therefore, this algorithm requires two reference points within the onset-offset search intervals where the onset/offset was assumed to be located between these points. This algorithm employs the polynomial approach, in which a line needs to be created between these two points. The farthest distance between the ECG segment and the line is considered an onset/offset point. The same polynomial approach is sequentially repeated for three possible segments. Heuristic analysis of the three possible pairs of points around each peak is done to select one out of the three possible locations as the final onset/offset point. Fig. 3 illustrates an example of QRS delineation using the polynomial lines for $\mathrm{Q}$ and $\mathrm{S}$ detection.

\subsection{Parameters extraction}

A new feature extraction approach of ECG represented by 26 parameters using Principal Component Analysis (PCA) and fiducial points detection methods is proposed. The statistical test ANOVA is used to analyze and determine the existence of statistically significant differences between the extracted ECG parameters in a resting state for both sitting and standing positions.

The 26 parameters that are extracted from the filtered ECG signal included the duration, time interval, amplitude of peak, and slope of the signals. These parameters are RR interval, PR interval, QRS duration, QT interval, QTc interval, P-wave duration, T-wave duration, PR segment, ST interval, ST segment, P ampli-

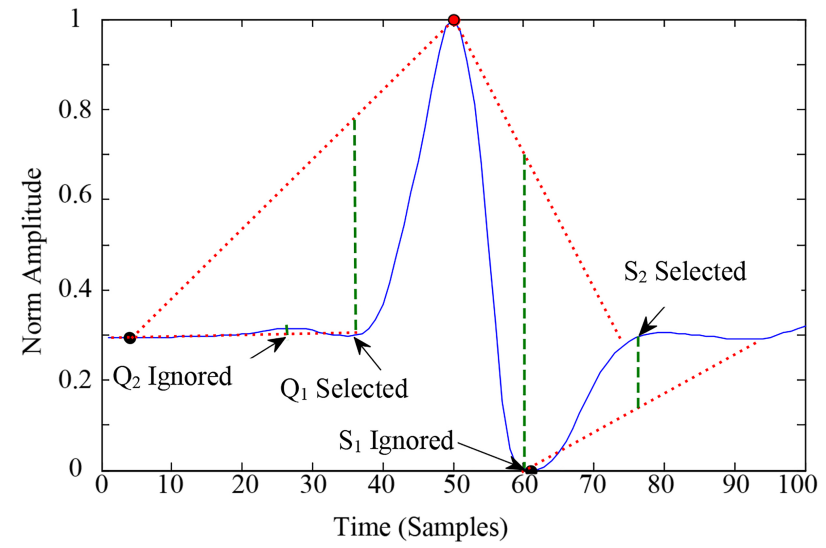

Fig. 3. Example of QRS complex delineation. The figure illustrates the heuristic search for candidate $\mathrm{Q}$ and $\mathrm{S}$ waves given the R-peak location. The green dash-lines represent the prominent candidate $\mathrm{Q}$ and $\mathrm{S}$ locations. Heuristic criteria are set to select the best-fit point among these locations.

tude, $\mathrm{R}$ amplitude, $\mathrm{T}$ amplitude, $\mathrm{Q}$ amplitude, $\mathrm{S}$ amplitude, RP Amp Ratio, RT Amp ratio, slope P1, slope P2, slope R1, slope $\mathrm{R} 2$, slope $\mathrm{T} 1$, slope $\mathrm{T} 2$, $\mathrm{P}$ slope ratio, $\mathrm{R}$ slope ratio, and $\mathrm{T}$ slope ratio. The ECG is recorded in the standing and sitting position, and values of various parameters are based on calculations made from both ECG recordings. In certain situations, such as exercise testing and evaluation, the ECG is also recorded in both of these positions. Thus, it becomes equally important to study and quantify the nature and magnitude of changes seen in the ECG with a change of posture from sitting to standing in healthy individuals.

\subsection{Statistical test}

Then statistical test, ANOVA, was conducted, and the outcome of the ANOVA test is presented in Table 2. In this case, the $P$ value is compared with the significance level to assess the null hypothesis. The null hypothesis states that the group of means is equal. Usually, a significance level $(\alpha)$ of 0.05 works well, where this level of 0.05 indicates a $5 \%$ risk of concluding that a difference exists when there is no actual difference. If $P$-value $\leq \alpha$, meaning the differences between some of the means are statistically significant and reject the null hypothesis.

Meanwhile, if the $P$-value $>\alpha$, then the differences between the means are not statistically significant. This study also used the Bonferroni procedure to group the information from pairwise comparisons of $t$-test with $95 \%$ confidence intervals (CIs). Bonferroni is chosen because the method is valid for equal or unequal sample size (Bland and Altman, 1995). The CI is used to determine the likely ranges for differences and to determine whether the differences are practically significant. The $95 \%$ CI indicates that it can be $95 \%$ confident that all the CIs contain true differences.

\section{Results}

The experimental hypothesis to evaluate whether a change in the subject position during the ECG recording alters the various components of the ECG curve is initiated by looking at the mean value of 26 parameters for a year observation. The data is divided into a group of weeks in which 1 week consists of data for 7 days. There are 16 weeks of sitting ECG data with 112 files. Fig. 4 il- 


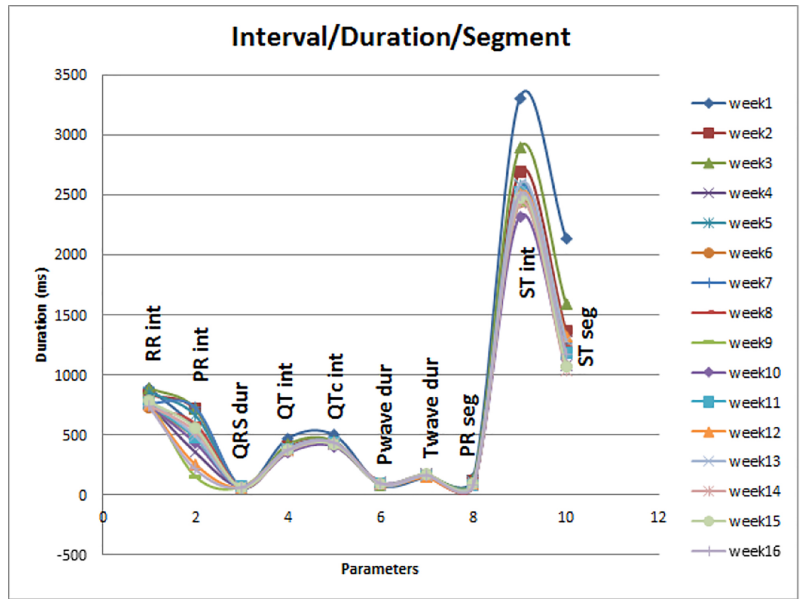

(a)

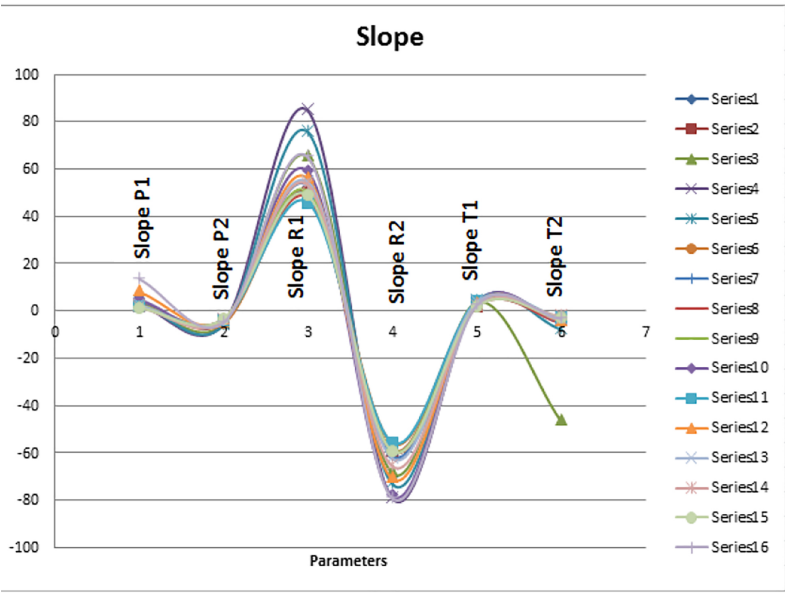

(c)

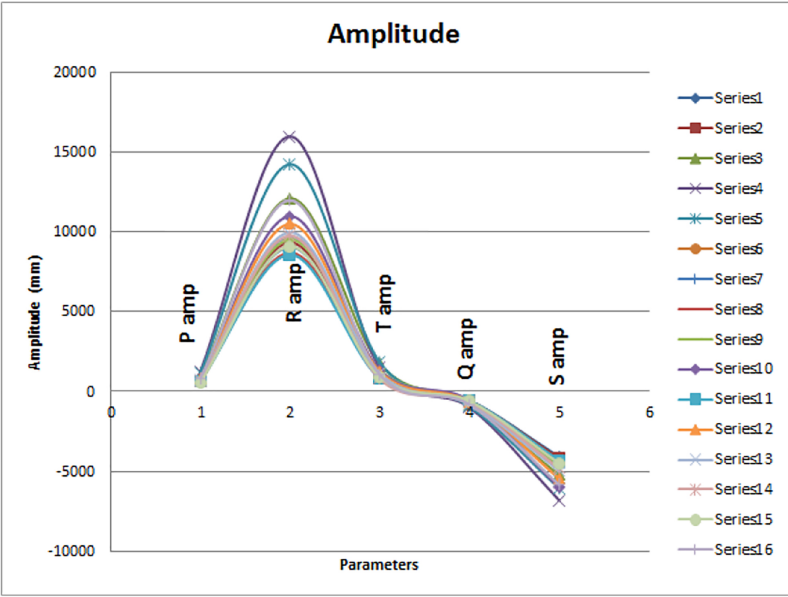

(b)

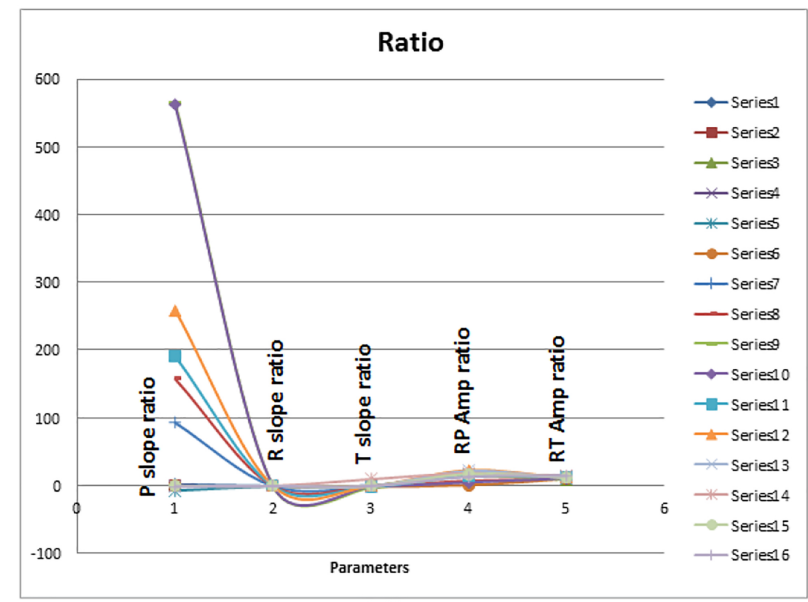

(d)

Fig. 4. A year observation of sitting 26 ECG parameters from Jan to Dec. (a) shows the time-domain related parameters. (b) amplitude parameters. (c) amplitude slope parameters. (d) amplitude slope ratio between two ECG components.

lustrated the significant changes of the 26 parameters of ECG for 16 weeks. The extracted set of 26 parameters for each ECG cycle is illustrated in Fig. 6.

Fig. 4 and 5 clearly show that the PR interval, ST interval, ST segment, R amplitude, Slope R1, Slope R2, and P slope ratio have different variations. Standing ECG, on the other hand, has about 18 weeks of data that were recorded and showed the same trend of different variations between the parameters. The one-way ANOVA is used then to determine whether there are any statistically significant differences between the means of three or more independent (unrelated) groups. Table 2 presents the statistical analysis results using ANOVA. The pairwise comparison between sitting and standing is calculated using a year data collection, which is about 454,400 cycles of sitting and 493,470 cycles of standing data. ANOVA is used to compare differences of means among the 2 groups by looking at variation in the data and where that variation. Specifically, it compares the amount of variation between groups with the amount of variation within groups.

Table 2 summarized the statistical ANOVA test results in terms of $P$-value and CI measure of the extracted 26 ECG parameters when comparing the sitting and standing positions. According to
Table 2, the highlighted rows are parameters that show significantly different between sitting and standing. A total of 10 parameters are reported with a significant difference where some of these parameters (i.e., PR int, PR seg, QT int, ST int, ST seg, and T wave dur) are clinically important parameters and any significant change of their values for the same subject could indicate a serious health problem. On the other hand, 16 ECG parameters show that sitting and standing are not significantly different. In other words, these parameters exhibit approximately similar behavior for both positions. Homogeneity of variances was violated, as assessed by Bonferroni's procedure for inequality of variances. A statistically significant difference shows from activity of sitting and standing at specific parameters, where (Table 3) simplified the results from the analysis.

Changes in body position are known to cause changes in the features of ECG, mainly in the intervals and segments, as shown in Table 3. These shifts have been attributed to the chest cavity, changes in lung volume, and change of electrode contact with the skin (Sigler, 1938). Most of the ECG wave amplitudes showed no significant difference between the sitting and standing position. Therefore, for specific purposes such as regular conditions 
Table 2. The pairwise CI (Bonferroni) of sitting and standing.

\begin{tabular}{|c|c|c|}
\hline Parameter & $P$-value & CI \\
\hline P amp & 0.76713 & $(-130.82019,59.16317)$ \\
\hline PR int & 0.02914 & $(7.50047,204.77463)$ \\
\hline PR Seg & 0.00000 & $(9.33298,21.63670)$ \\
\hline P slope ratio & 0.76189 & $(-336.01158,150.68840)$ \\
\hline $\mathrm{P}$ wave dur & 0.10773 & $(-4.61474,0.29611)$ \\
\hline Q amp & 0.00057 & $(55.82564,272.41974)$ \\
\hline QRS dur & 0.92800 & $(-1.35569,0.83339)$ \\
\hline QTc int & 0.97762 & $(-11.89285,16.33932)$ \\
\hline QT int & 0.00444 & $(4.71746,35.63593)$ \\
\hline $\mathrm{R}$ amp & 0.18808 & $(-1736.75144,216.48785)$ \\
\hline $\mathrm{RP}$ amp ratio & 0.87828 & $(-1.33737,2.43251)$ \\
\hline $\mathrm{RR}$ int & 0.00000 & $(38.74582,80.35886)$ \\
\hline $\mathrm{R}$ slope ratio & 0.03372 & $(0.00171,0.06275)$ \\
\hline $\mathrm{RT}$ amp ratio & 0.01006 & $(-1.75877,-0.16796)$ \\
\hline S amp & 0.37799 & $(-152.53813,657.10440)$ \\
\hline Slope P1 & 0.99922 & $(-0.43262,0.47874)$ \\
\hline Slope P2 & 0.40557 & $(-0.19580,0.79474)$ \\
\hline Slope R1 & 0.06203 & $(-10.72226,0.17529)$ \\
\hline Slope R2 & 0.22441 & $(-1.32967,9.01175)$ \\
\hline Slope T1 & 0.92556 & $(-0.58019,0.94970)$ \\
\hline Slope $\mathrm{T} 2$ & 0.84751 & $(-0.90849,0.47097)$ \\
\hline ST int & 0.00454 & $(34.23390,260.33508)$ \\
\hline ST seg & 0.00429 & $(65.50043,489.38892)$ \\
\hline $\mathrm{T}$ amp & 0.88980 & $(-129.06950,229.28805)$ \\
\hline $\mathrm{T}$ slope ratio & 0.86379 & $(-0.34745,0.18569)$ \\
\hline $\mathrm{T}$ wave dur & 0.02044 & $(-12.47187,-0.72235)$ \\
\hline
\end{tabular}

in monitoring the patient during the stress test exercise, recording ECG in stand position can be considered as a fair test. On the other hand, the RR interval shows significantly different during sitting and standing. An important contribution in this paper is that it can show that the HR for sitting is different from HR of standing for resting state. These parameters, RR interval, the PR interval, QT interval, ST interval, ST segment, PR segment, and T wave duration, also shows a significant difference between sitting and standing position, as these parameters are physiologically related to the frequency of HR. The results showed that 16 of the parameters specify that sitting and standing are not significantly different.

In contrast, another 10 parameters show that sitting and standing are significantly different from each other, as shown in Table 2. Fig. 5 illustrates the data distribution in Table 2, where the highlighted parameters (highlighted rows of Table 2) show a significant difference between sitting and standing. A change in the body position from standing to sitting results in changes in the ECG. These changes manifest in changes in the mean QRS, $\mathrm{P}$ wave, and $\mathrm{T}$ wave axis recorded in the frontal plane, as well as changes in the amplitude of the $\mathrm{P}, \mathrm{QRS}$, and T waveforms. ECG recordings in the sitting and standing positions are comparable with insignificant differences with specific parameters. The QTc interval shows a significant difference in the standing position compared to the sitting position and suggests changes in cardiac repolarization with the assumption of the standing position.

Several pathologies make it necessary to record an ECG from a patient in a vertical position, including neurological patients. This study demonstrates the way for a significant ECG analysis with using the lead-I in the absence of the possibility to conduct a classic ECG recording, due to a specific pathology (extensive burns, eczematous damage). This makes the findings of ECG analysis interesting in which some parameters (i.e., camel-hump patterns in lead- $\mathrm{I}^{1}$ ) are helpful for clinical interpretations and could be suggestive of neurologic injury or diagnosis of hypothermia patients. The results indicate that the electrocardiographically significant parameters obtained during such registration allow us to conclude both the processes of impulse conduction and the myocardial contractility to some extent.

\section{Discussion}

Employing ECG features in monitoring exercise progress could extend information in several aspects, for example, in detecting symptoms of heart disease and deficiency in the blood. Changes in the ECG resting as well as during and after exercises in healthy subjects were observed decades ago. However, a complete description of the whole ECG at all levels during or after exercise is needed for treatment of patients with neurologic injury from conditions such as stroke, cardiac arrest, and traumatic brain in-

\footnotetext{
1 National Institute for Health and Care Excellence. (2019) LeadI ECG devices for detecting symptomatic atrial fibrillation using single time point testing in primary care. Available at: https://ww w.nice.org.uk/guidance/dg35 (Accessed: 03 February 2020).
} 


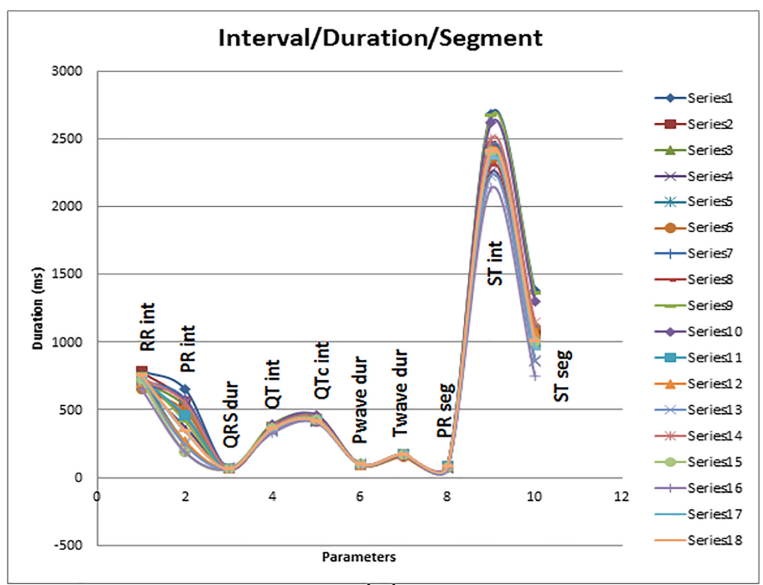

(a)

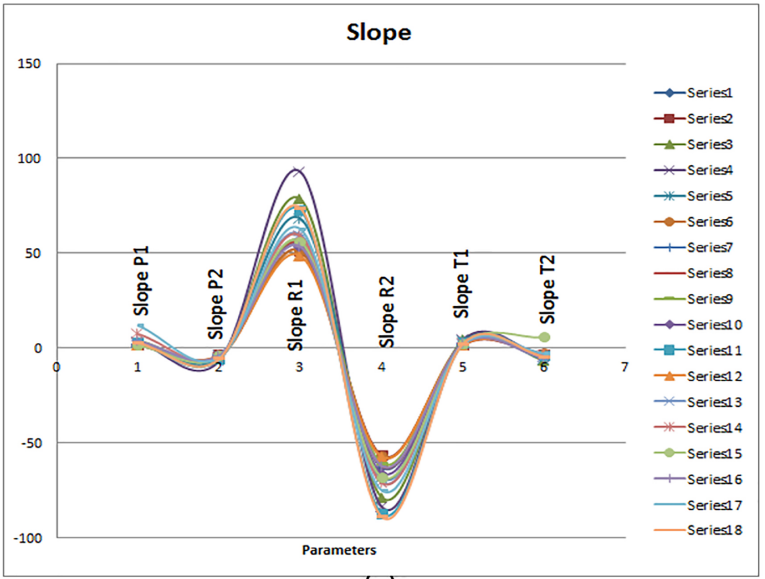

(c)

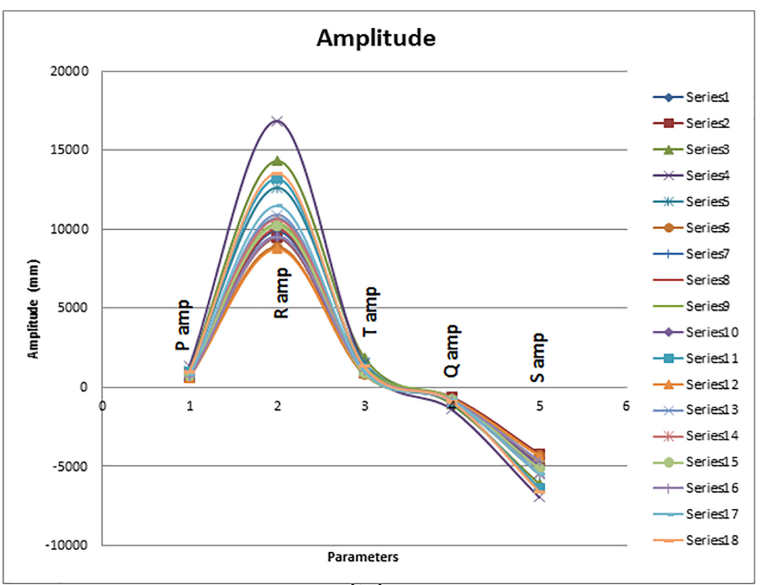

(b)

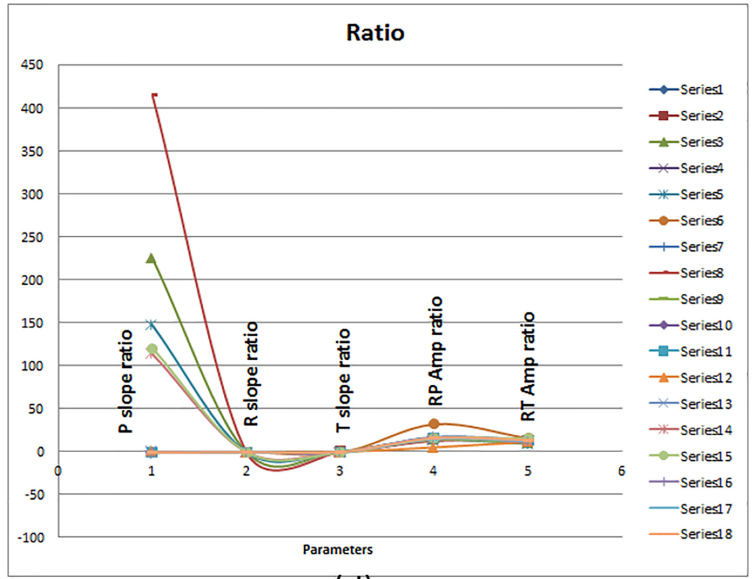

(d)

Fig. 5. A year observation of standing 26 ECG parameters from Jan to Dec. (a) shows the time-domain related parameters. (b) amplitude parameters. (c) amplitude slope parameters. (d) amplitude slope ratio between two ECG components.

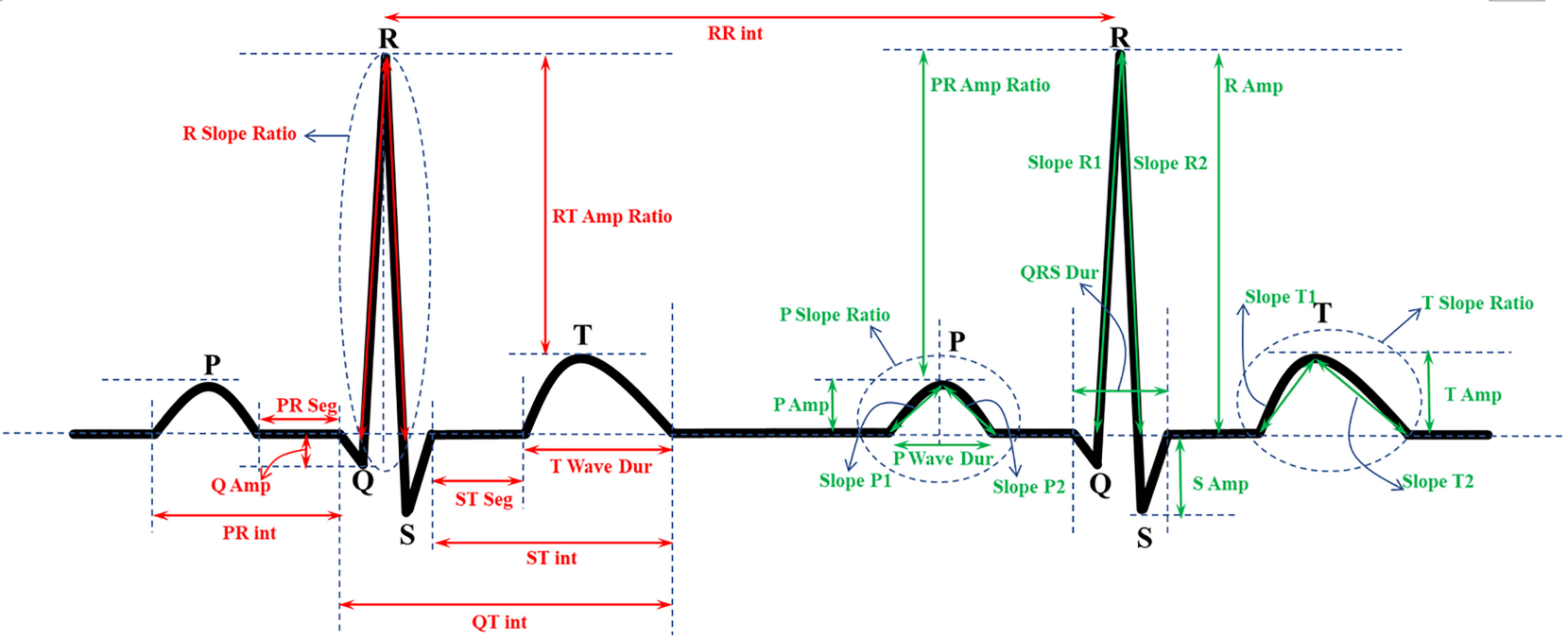

Fig. 6. The 26 parameters illustration. The figure consists of two subsequent cardiac cycles showing the measurement points of 10 time-domain, five amplitude, six amplitude slope, and five amplitude slope ratio parameters. A total of 25 parameters are extracted from each cardiac cycle, with one parameter (RR int) is shared between two contiguous cycles. 
Table 3. The division between significant differentiation of sitting and standing.

\begin{tabular}{lccc}
\hline Activity & Interval & Amplitude & Slope \\
\hline sit = stand & QTc interval, & R amplitude, & P slope ratio, \\
& QRS duration, & S amplitude, & T slope ratio, \\
& P wave duration & P amplitude, & Slope R1, \\
& & T amplitude, & Slope R2, \\
& RP amplitude ratio & Slope P1, \\
& & Slope P2, \\
& & Slope T1, \\
& & Slope T2 \\
\hline \multirow{2}{*}{ stand $\neq$ sit } & Q amplitude, & R slope ratio \\
& QT interval, & & \\
ST interval, & & \\
RR interval, & & \\
PR interval, & & \\
ST segment, & & \\
PR segment, & & \\
& T wave duration & & \\
\hline
\end{tabular}

jury. This article also contributes to improving the technique of computer processing of bioelectric signals, in the particular ECG, due to the qualitative description of the proposed procedure of the ECG delineation and parameters extraction when using the lead-I electrocardiogram.

Changes in body position are known to cause changes in the ECG, mainly in the electrical axis, QRS amplitude, ST segment, and $\mathrm{T}$ wave. These shifts have been attributed to changes in the anatomical orientation of the heart in the chest cavity, changes in lung volume, and change of electrode contact with the skin. Analysis performed on the 26 parameters using the pairwise t-test shows that 16 parameters $(62 \%)$ specify that sitting and standing are not significantly different, which means approximately similar; P wave duration, QRS duration, QTc interval, $\mathrm{P}$ amplitude, $\mathrm{S}$ amplitude, T amplitude, R amplitude, Slope P1, Slope P2, Slope T1, Slope T2, Slope R1, Slope R2, P slope ratio, T slope ratio, and $\mathrm{RP}$ amplitude ratio. Thus, these parameters are fair to be used as a marker for monitoring patients in an emergency room or diagnostic test in the laboratory on the position standing or sitting. Thus, it becomes equally important to study and quantify the nature and magnitude of changes seen in the ECG with a change of posture from sitting to standing in healthy individuals.

In most ECGs obtained in clinical practice, used digital processing algorithm built into the modern ECG system to assess the cardiac rhythm measures the HR, PR, QRS, and QT intervals. However, these studies are focused on a specific few parameters of the ECG. A recent review by Lyon et al. (2018) showed some ECG systems, which include; Sovilj et al. (2013) findings on the effect of simulated myocardial infarctions at various locations on ECG changes, specifically in the ST segment. Bacharova et al. (2016) used computer simulations to investigate the influence of left ventricular (LV) mass on QRS, and specifically increased QRS amplitude, in the context of LV hypertrophy. They also investigated the effect of slow ventricular activation on the QRS complex and showed how alteration of electrical properties might mimic ECG morphologies associated with anatomical abnormal- ities. Thamizhvani et al. (2018) monitors the abnormality of the heart by Electrocardiograph (ECG) waveform, which is formed of the PQRS pattern. Differentiation of the abnormalities based on the ECG signal can be applied for diagnosis in a clinical setting. They extracted the QRS complex to determine the presence of an arrhythmia. They showed that the QRS complex of an ECG signal plays a vital role in identifying the physical conditions of the heart.

\section{Ethics approval and consent to participate}

All data involved in this research are public datasets approved by the International Ethics Committee.

\section{Acknowledgments}

We are thankful to the authors of the freely available dataset used in this paper.

\section{Conflict of Interest}

The authors declare no conflict of interest.

Submitted: April 20, 2020

Received: May 16, 2020

Accepted: May 20, 2020

Published: September 30, 2020

\section{References}

Adams, M. G. and Drew, B. J. (1997) Body position effects on the ECG. Journal of Electrocardiology 30, 285-291.

Akhbari, M., Ghahjaverestan, N. M., Shamsollahi, M. B. and Jutten, C. (2018) ECG fiducial point extraction using switching Kalman filter. Computer Methods and Programs in Biomedicine 157, 129-136.

Akhbari, M., Shamsollahi, M. B., Jutten, C., Armoundas, A. A. and Sayadi, O. (2016) ECG denoising and fiducial point extraction using an extended Kalman filtering framework with linear and nonlinear phase observations. Physiological Measurement 37, 203-226.

Akhbari, M., Shamsollahi, M. B., Sayadi, O., Armoundas, A. A. and Jutten, C. (2016) ECG segmentation and fiducial point extraction using 
multi hidden Markov model. Computers in Biology and Medicine 79, 21-29.

Bacharova, L., Szathmary, V., Potse, M. and Mateasik, A. (2013) Computer simulation of ECG manifestations of left ventricular electrical remodeling. Journal of Electrocardiology 45, 630-634.

Bacharova, L., Szathmary, V., Svehlikova, J., Mateasik, A. and Tysler, M. (2016) QRS complex waveform indicators of ventricular activation slowing: Simulation studies. Journal of Electrocardiology 49, 790793.

Bashir, S., Bakhshi, A. D. and Maud, M. A. (2014) A template matchedfilter based scheme for detection and estimation of t-wave alternans. Biomedical Signal Processing and Control 13, 247-261.

Bland, J. M. and Altman, D. G. (1995) Multiple significance tests: the Bonferroni method. British Medical Journal 310, 170.

Bono, V., Mazomenos, E. B., Chen, T., Rosengarten, J. A., Acharyya, A., Maharatna, K., Morgan, J., M. and Curzen, N. (2014) Development of an automated updated Selvester QRS scoring system using SWTbased QRS fractionation detection and classification. IEEE Journal of Biomedical and Health Informatics 18, 193-204.

Castells-Rufas, D. and Carrabina, J. (2015) Simple real-time QRS detector with the MaMeMi filter. Biomedical Signal Processing and Control 21, 137-145.

Chatterjee, H. K., Gupta, R. and Mitra, M. (2012) Real time P and T wave detection from ECG using FPGA. Procedia Technology 4, 840-844.

Chen, C. and Chuang, C. (2017) A QRS detection and R point recognition method for wearable single-lead ECG devices. Sensors 17, 1969.

Citi, L., Brown, E. N. and Barbieri, R. (2012) A real-time automated pointprocess method for the detection and correction of erroneous and ectopic heartbeats. IEEE Transactions on Biomedical Engineering 59, 2828-2837.

Duskalov, I. K., Dotsinsky, I. A. and Christov, I. I. (1998) Developments in ECG acquisition, preprocessing, parameter measurement, and recording. IEEE Engineering in Medicine and Biology Magazine 17, 50-58.

Elgendi, M., Eskofier, B., Dokos, S. and Abbott, D. (2014) Revisiting QRS detection methodologies for portable, wearable, battery-operated, and wireless ECG systems. Plos One 9, e84018.

Hamilton, P. S. and Tompkins, W. J. (1986) Quantitative investigation of QRS detection rules using the MIT/BIH arrhythmia database. IEEE Transactions on Biomedical Engineering 33, 1157-1165.

Homaeinezhad, M. R., ErfanianMoshiri-Nejad, M. and Naseri, H. (2014) A correlation analysis-based detection and delineation of ECG characteristic events using template waveforms extracted by ensemble averaging of clustered heart cycles. Computers in Biology and Medicine 44, 66-75.

Ieong, C., Mak, P., Lam, C., Dong, C., Vai, M., Mak, P., Pun, S., Wan, F. and Martins, R. P. (2014) A 0.83- $\mu$ W QRS detection processor using quadratic spline wavelet transform for wireless ECG acquisition in 0.35- $\mu \mathrm{m}$ CMOS. IEEE Transactions on Biomedical Circuits and Systems 6, 586-595.

Karimipour, A. and Homaeinezhad, M. R. (2014) Real-time electrocardiogram P-QRS-T detection-delineation algorithm based on qualitysupported analysis of characteristic templates. Computers in Biology and Medicine 52, 153-165.

Khare, S. and Chawala, A. (2016) Effect of change in body position on resting electrocardiogram in young healthy adults. Nigerian Journal of Cardiology 13, 125.

Kim, J. and Shin, H. (2016) Simple and robust realtime QRS detection algorithm based on spatiotemporal characteristic of the QRS complex. Plos one 11, e0150144.

Kohler, B., Hennig, C. and Orglmeister, R. (2002) The principles of software QRS detection. IEEE Engineering in Medicine and Biology Mag- azine 21, 42-57.

Levkov, C., Mihov, G., Ivanov, R., Daskalov, I., Christov, I. and Dotsinsky, I. (2005) Removal of power-line interference from the ECG: a review of the subtraction procedure. Biomedical Engineering Online 4, 50.

Lin, C., Mailhes, C. and Tourneret, J. (2010) P- and T-wave delineation in ECG signals using a bayesian approach and a partially collapsed Gibbs sampler. IEEE Transactions on Biomedical Engineering 57, 2840-2849.

Lyon, A., Mincholé, A., Martínez, J. P., Laguna, P. and Rodriguez, B. (2018) Computational techniques for ECG analysis and interpretation in light of their contribution to medical advances. Journal of the Royal Society Interface 15, 20170821.

Madeiro, J. P. V., Nicolson, W. B., Cortez, P. C., Marques, J. A. L., Vázquez-Seisdedos, C. R., Elangovan, N., Ng, G. A. and Schlindwein, F. S. (2013) New approach for T-wave peak detection and T-wave end location in 12-lead paced ECG signals based on a mathematical model. Medical Engineering and Physics 35, 1105-1115.

Martínez, A., Alcaraz, R. and Rieta, J. J. (2010) Application of the phasor transform for automatic delineation of single-lead ECG fiducial points. Physiological Measurement 31, 1467-1485.

Martis, R. J., Acharya, U. R., Prasad, H., Chua, C. K. and Lim, C. M. (2013) Automated detection of atrial fibrillation using Bayesian paradigm. Knowledge-Based Systems 54, 269-275.

Merah, M., Abdelmalik, T. A. and Larbi, B. H. (2015) R-peaks detection based on stationary wavelet transform. Computer Methods and Programs in Biomedicine 121, 149-160.

Ning, X. and Selesnick, I. W. (2013) ECG enhancement and QRS detection based on sparse derivatives. Biomedical Signal Processing and Control 8, 713-723.

Pal, S. and Mitra, M. (2012) Empirical mode decomposition based ECG enhancement and QRS detection. Computers in Biology and Medicine 42, 83-92

Pan, J. and Tompkins, W. J. (1985) A real-time QRS detection algorithm. IEEE Transactions on Biomedical Engineering 32, 230-236.

Phukpattaranont, P. (2015) QRS detection algorithm based on the quadratic filter. Expert Systems with Applications 42, 4867-4877.

Sigler, L. H. (1938) Electrocardiographic changes occurring with alterations of posture from recumbent to standing positions. American Heart Journal 15, 146-157.

Sovilj, S., Magjarević, R., Lovell, N. H. and Dokos, S. (2013) A simplified 3D model of whole heart electrical activity and 12-lead ECG generation. Computational and Mathematical Methods in Medicine 2013 $1-10$.

Tafreshi, R., Jaleel, A., Lim, J. and Tafreshi, L. (2014) Automated analysis of ECG waveforms with atypical QRS complex morphologies. Biomedical Signal Processing and Control 10, 41-49.

Thamizhvani, T. R., Joseph, J. E., Babu, B., Rithikka, U., Rohini, D. and Dhivya, A. J. (2018) Comparison of normal and abnormal conditions in ECG using RR variability and spectral density. International Journal of Engineering and Technology 7, 28.

Yochum, M., Renaud, C. and Jacquir, S. (2016) Automatic detection of P, QRS and T patterns in 12 leads ECG signal based on CWT. Biomedical Signal Processing and Control 25, 46-52.

Zhu, H. and Dong, J. (2013) An R-peak detection method based on peaks of Shannon energy envelope. Biomedical Signal Processing and Control 8, 466-474.

Zidelmal, Z., Amirou, A., Adnane, M. and Belouchrani, A. (2012) QRS detection based on wavelet coefficients. Computer Methods and Programs in Biomedicine 107, 490-496.

Zidelmal, Z., Amirou, A., Ould-Abdeslam, D., Moukadem, A. and Dieterlen, A. (2014) QRS detection using S-Transform and Shannon energy. Computer Methods and Programs in Biomedicine 116, 1-9. 